\title{
Game Theoretic Rate Adaptation for Spectrum-Overlay Cognitive Radio Networks
}

\author{
Laxminarayana S Pillutla and Vikram Krishnamurthy \\ Department of Electrical and Computer Engineering \\ The University of British Columbia \\ 2332 Main Mall, Vancouver, BC V6T1Z4, Canada. \\ E-mail:\{laxp, vikramk\}@ece.ubc.ca
}

\begin{abstract}
We consider the issue of fair share of the spectrum opportunity for the case of spectrum-overlay cognitive radio networks. Owing to the decentralized nature of the network, we adopt a pricing based game-theoretic approach where the actions of players would be choosing the modulation rate. The resulting game can be verified to be supermodular, and thus has atleast one pure strategy Nash equilibrium. Next, we propose a Stochastic Approximation based algorithm for the computation of best response correspondence whose convergence to the Paretodominant Nash equilibrium can be established. Furthermore, we also propose a decentralized algorithm for tuning the price factor of the network. Our simulation results demonstrate the gains that can be achieved with pricing.
\end{abstract}

\section{INTRODUCTION}

Cognitive Radio (CR) is envisioned as a key technology to address the problem of spectrum underutilization in wireless networks. A cognitive wireless transceiver can adapt its operating frequency, power level, and bandwidth of transmission in response to the changes in its environment [1]. The ability to adapt the former most parameter (i.e., the operating frequency) is commonly referred to in the literature as Dynamic Spectrum Access (DSA). DSA allows for spectrum sharing between secondary (unlicensed) users and primary (licensed) users without compromising on the privileges of the primary user. DSA can be achieved broadly using two approaches: spectrumunderlay and spectrum-overlay [2] which are described next.

In the spectrum-underlay approach the secondary users access the spectrum by operating below the power level of the primary user. The power levels of the secondary users are determined by the Interference-temperature (or a variant of it) constraint, imposed by the spectrum regulators. Consequently, the issue of secondary user power control (or regulation) is very important and has received a lot of attention from the research community [3] [4].

In a spectrum-overlay approach the secondary users access the spectrum opportunistically i.e., whenever the primary users are inactive. However since the collisions with the primary user are inevitable, hence the access schemes are to be designed so that the probability of collision with the primary user is below a certain limit [5].

In this paper we focus on the ad hoc spectrum-overlay $\mathrm{CR}$ networks and address the issue of regulating the channel occupancy of secondary users. This is of great importance in the spectrum-overlay networks, where the secondary users may get a chance to transmit occasionally, and hence there is a greater need for equal distribution of the spectrum opportunity.

We employ pricing as a means to ensure fair distribution of the spectrum opportunity. Pricing is a common technique employed to achieve a socially desirable result. In this paper pricing is employed to control the modulation rate of the users of the network. The objective of every secondary user of the network is to maximize its utility, which is equal to the difference between the average throughput a secondary user would obtain for transmitting at a specific rate, and a cost that is proportional to the fraction of time the user occupies the channel. The cost is incurred due to the price imposed on the user for channel occupation. The actions of the secondary users is to select an appropriate rate for transmission. The resulting game is analyzed under the Non-Cooperative GameTheoretic framework.

\section{A. Main Results}

1) We prove the pricing based non-cooperative rate adaptation game to be a supermodular game (under a fairly practical sufficient condition). Because of this the game always has atleast one pure strategy Nash equilibrium $(N E)$ and is contained in a set bounded by the smallest and largest NE [6] [7].

2) We prove that the smallest and largest NE are nondecreasing functions of the price factor. We also prove the smallest NE to be Pareto-dominant.

3) We propose a Stochastic Approximation (SA) based algorithm which is used by the players to compute the Best Response Correspondence. The algorithm converges almost surely to the smallest Nash equilibrium. We also propose an algorithm for price factor adaptation that is especially attractive for decentralized wireless networks.

\section{B. Paper Organization}

The rest of this paper is organized as follows: Section II contains system model description of the spectrum-overlay CR network and assumptions. Section III contains the formulation of the non-cooperative rate adaptation game. Section IV contains analysis of the non-cooperative game formulated in 
Section III. Section V contains numerical simulation results. Section VI contains conclusions.

\section{Spectrum-Overlay CR Network Model and ASSUMPTIONS}

A. System model description of a spectrum-overlay CR wireless network

Consider an $N_{s}$ secondary user wireless network trying to access a channel designated for exclusive use of primary users opportunistically. The primary user activity on the channel is assumed to be an ON-OFF model alternating between $\mathrm{ON}$ and OFF states. Once an OFF state is observed by a secondary user it uses a portion of OFF period for its transmission. We model the sojourn time of the $\mathrm{ON}$ and OFF states as an exponential random variable with probability density function(s) (pdf) $\frac{1}{T_{O N}} \exp \left(-\frac{x}{T_{O N}}\right), \frac{1}{T_{O F F}} \exp \left(-\frac{y}{T_{O F F}}\right)$ respectively, where $T_{O N}$ and $T_{O F F}$ denote the mean ON and OFF time of primary users. One can use other distributions such as Erlang, Pareto etc., to model the sojourn times of the $\mathrm{ON}$ and OFF states. The value of $T_{O N}$ and $T_{O F F}$ can be obtained from spectrum activity measurements.

For transmission of each packet we assume the secondary users employ a random access mechanism with probability (q) equal to $1 / N_{s}$. This is a fairly reasonable assumption, for example, the distributed coordination function (DCF) in IEEE 802.11 standard ensures that all the users in the network transmit with a uniform probability value in the long run [8].

We further assume the secondary users are capable of changing their transmission rate by varying their modulation scheme on a given channel. The choice of modulation scheme is decided by the transmitter upon obtaining channel quality information from the receiver during the request to send (RTS)/ clear to send (CTS) mechanism used commonly in ad hoc networks. We assume the wireless channel to be of Rayleigh fading type corrupted by an additive white Gaussian noise. The probability of bit error in such a channel with Quadrature Amplitude Modulation (QAM) (with a square constellation approximation) can be written as [9]

$$
\begin{aligned}
p_{b}(b \mid x) & =Q(\sqrt{2 b x}) \text { for }(b=1) \\
& =\frac{4}{b}\left(1-\frac{1}{2^{\frac{b}{2}}}\right) Q\left(\sqrt{\frac{3 b x}{2^{b}-1}}\right) \text { for }(b \geq 2),
\end{aligned}
$$

where $b$ denotes the modulation rate (i.e., the number of bits transmitted per symbol), $\bar{\gamma}$ denotes the mean channel SNR, and $\gamma$ denotes the instantaneous channel SNR. The error probability expression in Equation (1) will be useful in the next section where we formulate the utility expression for each node. We note that the game-theoretic analysis in this paper holds for general distributions such as Ricean, Nakagami, and also to the case when the fading process evolves according to a Markov chain. The stochastic approximation used in Algorithm 1 in Section IV B is valid for all the above mentioned cases.
We remark that in our work, the secondary users adapt their modulation rate to vary their transmission rate $(R)$ equal to $W b$, where $W$ is the symbol rate.

\section{B. Notation}

Throughout this paper, we use $\mathbb{R}^{n}$ to denote an $n$ dimensional Euclidean space, and use $\mathrm{x}$ (i.e., lower case bold faced letters) to denote a vector that belongs to the set $X \subseteq \mathbb{R}^{n}$. We use the standard game-theoretic notation $u_{i}\left(b_{i}, b_{-i}\right)$ to indicate the dependence of the utility function on $i^{\text {th }}$ player's action $b_{i}$, and on other player's actions denoted as $b_{-i}$ i.e., $b_{-i} \triangleq\left[b_{1}, b_{2}, \cdots, b_{i-1}, b_{i+1}, \cdots, b_{N}\right]$. Occasionally we will use the notation $u_{i}(\mathbf{b})$ to denote the outcome of the game in terms of the selected actions of all players i.e., $\mathbf{b} \triangleq\left[b_{1}, b_{2}, \cdots, b_{i}, \cdots, b_{N}\right]$. We use " $\times$ " to denote the cartesian product of sets, and $\mathbb{E}_{x}$ to denote the mathematical expectation with respect to the random variable $x$. We assume the partial ordering in our paper to be " $\geq$ ". Also if we define $\mathbf{x} \triangleq\left[x_{1}, x_{2}, \cdots, x_{N}\right]$ and $\mathbf{y} \triangleq\left[y_{1}, y_{2}, \cdots, y_{N}\right]$, then $\mathbf{x} \geq \mathbf{y}$ implies $x_{i} \geq y_{i}$ for all $x_{i}, y_{i}$ respectively.

\section{iII. Formulation of the Non-Cooperative Rate ADAPTATION GAME}

\section{A. utility function}

A utility function assigns numerical value to the elements of the action set. In any network a rational and selfish node aims to maximize its (expected) throughput. The selfish part of the utility is thus given by

$$
u_{j, \text { benefit }}\left(b_{j}\right)=r \mathbb{E}_{\gamma}\left(f\left(b_{j}\right)\right),
$$

where $r=\left(T_{O F F} /\left(T_{O N}+T_{O F F}\right)\right)$ denotes the probability that the channel is free from primary user activity, $b_{j}$ denotes the rate, $f($.$) denotes a general transport layer throughput$ expression that depends on the modulation rate $b_{j}$ and the packetsize $M_{j}$ used by the node on a given channel, and is defined as in Eq. (3) below for an UDP based traffic. The expectation in Eq. (2) is with respect to the channel SNR $\gamma$.

For the User Datagram Protocol (UDP), the throughput expression can be written as

$$
f\left(b_{j}\right)=\left(1-\frac{\delta_{j}}{M_{j}}\right) W_{j} b_{j}\left[1-p\left(b_{j} \mid \gamma\right)\right],
$$

where $p\left(b_{j} \mid \gamma\right)$ is the packet loss probability which is computed below, $W_{j}$ is the symbol rate (expressed in symbols per second (sps)) of user $j$ and all the other variables are as defined above.

In general wireless networks packet losses occur only due to channel errors. However in a spectrum-overlay CR network the packet losses can also occur due to service interruption from a primary user. The probability of service interruption on a given channel $\left(p_{\mathrm{SI}}\right)$ can be written as the probability that the OFF period on that channel is less than the packet transmission time which is equal to $M_{j} /\left(W_{j} b_{j}\right)$ i.e.,

$$
\begin{aligned}
p_{\mathrm{SI}} & =P\left(t_{\mathrm{OFF}}<M_{j} /\left(W_{j} b_{j}\right)\right) \\
& =\left(1-\exp \left(-M_{j} /\left(W_{j} b_{j} T_{\mathrm{OFF}}\right)\right)\right)
\end{aligned}
$$


The packet loss probability due to channel errors under the assumption that each bit in the packet gets corrupted independently can be written as

$$
p_{\mathrm{CH}}\left(b_{j} \mid \gamma\right)=1-\left(1-p_{b}\left(b_{j} \mid \gamma\right)\right)^{M_{j}}
$$

where $p_{b}($.$) is the probability of bit error defined as in Eq.$ (1). The probability of collision $p_{\mathrm{CO}}$ due to contention among secondary users can be written as $p_{\mathrm{CO}}=1-q(1-q)^{N_{s}-1}$, where $q=1 / N_{s}$ as mentioned before.

The total packet loss probability on a given channel can therefore be written as follows:

$$
p\left(b_{j} \mid \gamma\right)=1-\left(1-p_{\mathrm{SI}}\right)\left(1-p_{\mathrm{CH}}\right)\left(1-p_{\mathrm{CO}}\right),
$$

where $p_{\text {SI }}$ is the probability of service interruption from the primary user on a given channel, and $p_{\mathrm{CH}}$ is the loss probability due to channel errors given by Eq. (5) and $p_{\mathrm{CO}}$ is the packet collision probability as defined above.

In wireless networks equipped with a base station, the base station would schedule the secondary users so that each user may get a fair share to transmit on the channel. However in the absence of a base station, a secondary user may employ smaller rate to prolong its transmission time, and hence increase its throughput. To desist such a selfish behavior on the part of secondary users and ensure fair share of the spectrum opportunity to all the secondary users we impose a price on every secondary user for accessing the channel that is proportional to its channel occupancy. In most economic applications pricing is employed to generate revenue for a system, however here pricing is used to motivate the users to adopt a social behavior. The cost incurred by the $j^{\text {th }}$ user due to pricing can be written as

$$
u_{j, \mathrm{cost}}=\lambda\left(\frac{\frac{M_{j}}{W_{j} b_{j}}}{\frac{M_{j}}{W_{j} b_{j}}+\sum_{i \neq j} \frac{M_{i}}{W_{i} b_{i}}}\right),
$$

where $\lambda$ is the "price" the $j^{\text {th }}$ user has to pay for access on a given channel $l$. The pricing scheme adopted can in general be of any form, however we restrict ourselves to a linear pricing scheme, wherein the price increases monotonically with an increase in access time of the user on a given channel.

The net utility incurred by the $j^{\text {th }}$ user (in bps) is the difference between the total benefit (cf. Equation (2)), and the total cost (cf. Equation (7)), therefore

$$
\begin{aligned}
& u_{j}(\mathbf{b})=u_{j, \text { benefit }}-u_{j, \text { cost }} \\
& =r \mathbb{E}_{\gamma}\left(f\left(b_{j}\right)\right)-\lambda\left(\frac{\frac{M_{j}}{W_{j} b_{j}}}{\frac{M_{j}}{W_{j} b_{j}}+\sum_{i \neq j} \frac{M_{i}}{W b_{i}^{l}}}\right),
\end{aligned}
$$

where $\mathbf{b}$ denotes the vector comprising of rates of all the users as mentioned in our notation.

Using the utility function in Equation (8) one can formulate a non-cooperative game where the actions of players would be choosing the rate $b_{j}$ on a given channel. The rules of such a game are described in the next subsection.

\section{B. Rules of the NRSGP}

In this subsection we formulate the Non-Cooperative Rate Selection Game with Pricing (NRSGP).

Let $G_{\text {NRSGP }}=\left[\mathcal{N},\left\{B_{j}\right\},\left\{u_{j}\right\}\right]$ denote the NRSGP where $\mathcal{N}=\left\{1,2, \cdots, N_{s}\right\}$ denote the set of secondary users in the ad hoc network, $B_{j}$ is the strategy set of user $j$, and $u_{j}$ defined in Equation (8) is their utility function. In an NRSGP each user $j$ selects a rate $b_{j}$ such that $b_{j} \in B_{j}$, where $B_{j}$ is the user strategy set defined as in Equation (9) below.

The strategy set of user $j$ in an NRSGP is the cartesian product of compact subsets of $\mathbb{R}$ i.e.,

$$
B_{j} \triangleq B_{j} \triangleq\left\{b_{j, \min }, \cdots \cdots, b_{j, \max }\right\},
$$

where $b_{j, \min }>0$ denotes the minimum rate at which a secondary user intends to transmit. The value of $b_{j, \max }$ is determined by the peak power of the secondary user battery source.

In an NRSGP every secondary user before transmission of a packet determines its rate by maximizing its utility in a distributed fashion. Formally, the distributed optimization problem can be stated as follows:

$$
\begin{aligned}
& \text { (NRSGP) } \max _{b_{j}} u_{j}\left(b_{j}, b_{-j}\right), \\
& \text { s.t. } b_{j} \in B_{j} \text { for all } j \in \mathcal{N},
\end{aligned}
$$

where $u_{j}(.,$.$) is given by Equation (8) and B_{j}$ is defined as in Equation (9).

Our aim is to determine if an equilibrium (or stable point) exists in case the game is played repeatedly by all the secondary users of the network, the analysis of which is presented next.

\section{AnAlysis of NRSGP - A Supermodular GAme APPROACH}

In this section we analyze the Non-Cooperative Rate Selection Game with Pricing (NRSGP) using supermodular game theory.

\section{A. NRSGP - Supermodular Game}

Theorem 1: The following results are true with respect to the game $G_{\mathrm{NRSGP}} \triangleq\left[\mathcal{N},\left\{B_{j}\right\},\left\{u_{j}\right\}\right]$ defined in Eq. (10):

(i). The game is supermodular if $b_{j, \min }$ in Eq. (9) is chosen so that $b_{j, \min } \geq \frac{\frac{M_{j}}{W_{j}}}{\sum_{i \neq j} \frac{M_{i}}{W_{i} b_{i}}}$.

(ii). The game has atleast one pure strategy Nash equilibria. The set of Nash equilibria are contained within the set $\left(\mathrm{NE}_{\lambda}\right)$ defined as follows: $\mathrm{NE}_{\lambda}=$ $\left\{\mathbf{b}(\lambda) \in B \mid \mathbf{b}_{S}(\lambda) \leq \mathbf{b}(\lambda) \leq \mathbf{b}_{L}(\lambda)\right\}$, where $B \triangleq \times_{j=1}^{N_{s}}$ denotes the joint strategy space of the game $G_{\text {NRSGP. }}$

(iii). The smallest and largest $\mathrm{NE}$ in the set $\left(\mathrm{NE}_{\lambda}\right)$ namely $\mathbf{b}_{S}(\lambda)$ and $\mathbf{b}_{L}(\lambda)$ are non-decreasing functions of $\lambda$, the price.

(iv). The smallest $\mathrm{NE} \mathbf{b}_{S}(\lambda)$ is the Pareto-dominant equilibrium i.e., no equilibrium other than $\mathbf{b}_{S}(\lambda)$ in the set of $\mathrm{NE}_{\lambda}$ would yield higher utilities for all users. 
Proof: The part (i) can be easily verified using the Theorem 4 of [7]. Part (ii) follows from the Theorem 5 of [7]. Part (iii) follows from the Theorem 6 of [7]. Part (iv) follows from the basic definition of NE and by noting that the utilities of all the players in the game are decreasing in other players actions.

\section{B. Algorithm for the Computation of Best Response (BR) Correspondence}

The following Stochastic Approximation (SA) based algorithm is used by every secondary user of the network to compute the BR correspondence.

Algorithm 1 Algorithm for computation of best response using stochastic approximation (SA).

1: At any update instant $k$ the $j^{\text {th }}$ user computes its best response correspondence $\mathcal{B R}_{j}^{(k)}\left(b_{-j}^{(k-1)}\right)$ as below:

a. Compute the $b_{j, \min }$ of $j^{\text {th }}$ user strategy space $B_{j}$ as follows:

$$
b_{j, \min }=\frac{\frac{M_{j}}{W_{j}}}{\sum_{i \neq j} \frac{M_{i}}{W_{i} b_{i}^{(k-1)}}}
$$

where $\frac{M_{i}}{W_{i} b_{i}^{(k-1)}}$ for all $i \neq j$ such that $i \in \mathcal{N}$ in practice can be obtained via the RTS frame broadcasted previously by all $i$ as noted before (cf. Remark 1 below Theorem 1).

b. Using the independent SNR estimates $\gamma_{q}(q=$ $1,2, \cdots, Q)$ obtain an estimate of the utility function $\hat{u}_{j}\left(b_{j}, b_{-j}^{(k-1)}\right)$ for each $b_{j} \in B_{j}$ as below:

$$
\begin{array}{r}
\hat{u}_{j}\left(b_{j}, b_{-j}^{(k-1)}\right)=\frac{1}{Q} \sum_{q=1}^{Q}\left(1-\frac{\delta_{j}}{M_{j}}\right) W_{j} b_{j}\left(1-p\left(b_{j} \mid \gamma_{q}\right)\right)^{M_{j}} \\
-\lambda\left(\frac{\frac{M_{j}}{W_{j} b_{j}}}{\frac{M_{j}}{W_{j} b_{j}}+\sum_{i \neq j} \frac{M_{i}}{W_{i} b_{i}^{(k-1)}}}\right)
\end{array}
$$

where $\lambda$ denotes the price imposed on each user for channel occupancy.

c. Assign $\mathcal{B R}_{j}^{(k)}()=.\underset{b_{j} \in B_{j}}{\arg \max } \hat{u}_{j}($.$) , where \hat{u}_{j}$ is defined as in equation above.

2: Assign the rate $b_{j}^{(k)}=\min \left(\mathcal{B R}_{j}^{(k)}().\right)$, in case if the best response correspondence $\mathcal{B} \mathcal{R}_{j}^{(k)}($.$) is not unique.$

Note that at each update instant $k$, a given user $i$ uses an estimate $\hat{u}_{i}$ of its utility function to compute its best response correspondence $\mathcal{B R}_{i}^{(k)}($.$) . The estimate is computed$ by using the independent channel SNR estimates $\gamma_{q}$ (for $q=1,2, \cdots, Q)$. This is necessary because the utility function of each user namely $u_{i}$ cannot be computed analytically.

The Algorithm 1 can be proved to converge to the smallest Nash equilibrium $\left(\mathbf{b}_{S}(\lambda)\right)$ almost surely provided it starts at the smallest point of the joint strategy space $\times_{j=1}^{N_{s}} B_{j}$. This follows from the Theorem 4.3.1 in [6]. Due to lack of space we omit it.

\section{Price vector $\lambda$ adaptation}

The following algorithm can be used to tune the price vector by the secondary users without a pricing controller.

Algorithm 2 Algorithm to tune the price $\lambda$ of secondary users.

1: At the start all the secondary users set $\lambda=0$.

2: The secondary users determine their rates according to Algorithm 1.

3: The secondary users increment $\lambda$ as $\lambda=\lambda+\Delta$.

4: If any secondary user reports decrease in utility the users stop price increment, else go to step 3 and continue.

\section{Simulation Results}

We set the frame size $M_{i}$ of all users $i=1,2, \cdots, N_{s}$ equal to 2000 bytes. We assume the combined bit overhead $\delta_{i}, i=1,2, \cdots, N_{s}$ of all users as equal to 100 bytes. This implies the actual payload is equal to 1900 bytes. The symbol rate $W_{i}, i=1,2, \cdots, N_{s}$ of all the users is set equal to $10^{6}$ symbols per second. The maximum rate $b_{i, \max }$ of all the users is set equal to 20 . We set the mean channel SNR values for users (1-5) as 10,15, 20, 25, 30 respectively. We assume the fading in the channel to be Rayleigh distributed. Therefore, the received per bit SNR $\gamma$ has an exponential distribution. We assume the mean OFF time of the primary user to be 0.1 seconds and the primary user load factor $(A)=T_{O N} /\left(T_{O N}+\right.$ $T_{O F F}$ ) to be 0.2 (which is higher than the spectral activity measurements reported for most bands, hence would make our analysis conservative), which implies the $T_{O F F}$ to be equal to 0.025 seconds.

For the case of Fig. 1 we first get the equilibrium rates for the case when the price factor $\lambda=0$. This corresponds to the case where there is no price imposed on a user for channel occupancy. Once the equilibrium is attained in this case (which is guaranteed by our analysis in Section IV-A), all the nodes in the network will increment their price factor as noted in Algorithm 2, and converge to the corresponding equilibrium. The price increment is continued until one of the user (which is user 1 in our case since it has the lowest mean channel SNR) reports reduction in utility. The corresponding price $\lambda_{\mathrm{B}}$ is indeed the best for the given network scenario.

The Fig. 1 shows the plot of equilibrium rates of various users on a given channel versus the SNR. The figure shows that an increase in price $\lambda$ forces the users to adopt higher rates as proved in Theorem 1, where we proved the smallest and largest equilibrium to be non-decreasing with respect to the price $\lambda$ (note that the Algorithm 1 attains the smallest equilibrium almost surely). Also notice that the increase in rate with respect to the price is significant at lower SNR compared to that at high SNR. This is to be expected since the price may not be too large for the users to increase their rates drastically.

We next present simulation results on the impact of pricing on the overall network throughput. To this end we consider a 


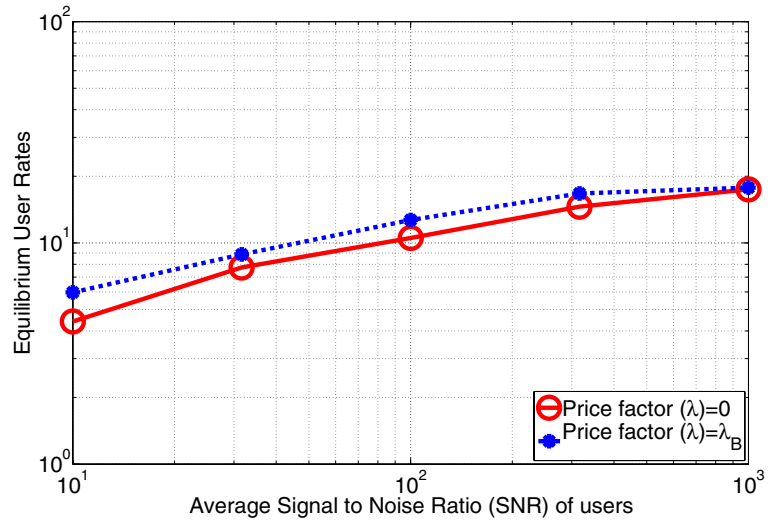

Fig. 1. The figure shows the plot of user rates versus the average SNR. The plot shows that with an increase in price $(\lambda)$ the rate of each user increases as proved in part (iii) of Theorem 1.

worst case scenario in WLANs. A typical worst case scenario contains large number of low-rate users and a smaller number of moderate and high rate users. The aforementioned scenario is worst from the overall network throughput perspective, since the low rate users occupy the channel for a long time and deny the moderate and high rate users of their fair share to transmit on the channel. Accordingly we assume there are 5 users with average SNR equal to $10 \mathrm{~dB}, 3$ users with average SNR equal to $15 \mathrm{~dB}$ and 2 users with average SNR equal to $30 \mathrm{~dB}$. To mimic the DCF mechanism used in WLANs we assume each user before the transmission of a frame accesses the channel with a probability equal to 0.1 . This choice of access probability ensures all the users obtain a fair share to transmit on the channel. We assume each user transmits at a rate $b_{i}$. The rate $b_{i}$ is chosen equal to the equilibrium rates. The equilibrium rates for the current simulation scenario are obtained by using the same procedure described for Fig. 1 above (i.e., we first assume there is no price $(\lambda=0)$, allow the system to converge to the equilibrium point, then increase the price and allow it to converge to the corresponding equilibrium point. The procedure is continued until at least one user reports a reduction in its equilibrium utility).

Figure 2 shows the plot of total network throughput with and without pricing. The figure shows that the total network throughput increases significantly due to pricing. This can be attributed to an increase in occupancy level for the $15 \mathrm{~dB}$ and $30 \mathrm{~dB}$ users which can be attributed to an increase in rate of the $10 \mathrm{~dB}$ users due to pricing as observed in Fig. 1.

\section{CONCLUSIONS}

We considered pricing based non-cooperative game theory based framework to ensure fair share of the spectrum opportunity in a spectrum-overlay $\mathrm{CR}$ wireless network. The resulting game was proved to be supermodular and hence has atleast one pure strategy Nash equilibrium in a set bounded by the smallest and largest NE. The smallest and largest $\mathrm{NE}$ are shown to be non-decreasing functions of the price

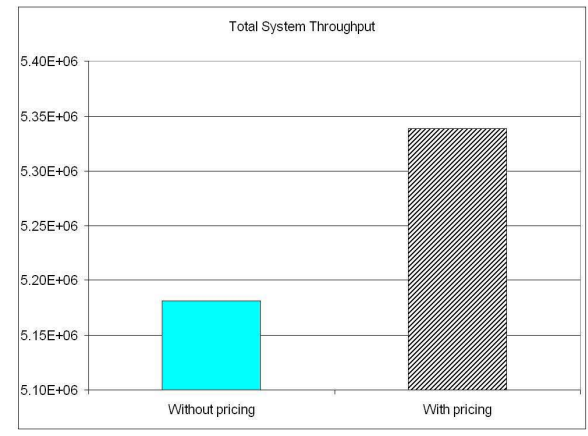

Fig. 2. The figure shows the total network throughput for the case of with and without pricing. The figure shows that an appropriate choice of price can lead to higher overall network throughput.

factor. Further the smallest NE was shown to be Paretodominant. Next, we proposed stochastic approximation based algorithm to compute the best response whose convergence to the smallest $\mathrm{NE}$ was established. We also proposed an algorithm for the tuning of price factor of the network.

\section{REFERENCES}

[1] S. Haykin, "Cognitive Radio: Brain-Empowered Wireless Communications," IEEE Journal on Selected Areas in Communications, vol. 23, no. 2, pp. 201-220, Feb. 2005.

[2] Q. Zhao and B. M. Sadler, "A Survey of dynamic Spectrum Access: Signal Processing, Networking, and Regulatory Policy," IEEE Signal Processing Magazine, vol. 24, no. 3, pp. 79-89, May 2007.

[3] J. Huang, R. A. Berry, and M. L. Honig, "Auction-Based Spectrum Sharing," Springer, Mobile Networks and Applications, vol. 11, no. 3, pp. 405-418, April 2006.

[4] F. Wang, M. Krunz, and S. Cui, "Spectrum Sharing in Cognitive Radio networks," Technical Report TR-UA-ECE-2007-1, University of Arizona, July 2007.

[5] Q. Zhao, L. Tong, A. Swami, and Y. Chen, "Decentralized Cognitive MAC for Spectrum Access in Ad Hoc Networks: A POMDP Framework," IEEE Journal on Selected Areas in Communications, vol. 25, no. 3, pp. 589-600, April 2007.

[6] D. M. Topkis, Supermodularity and Complementarity, Princeton University Press, New Jersey, 1998.

[7] P. Milgrom and J. Roberts, "Rationalizability, Learning, and Equilibrium in Games with Strategic Complementarities," Econometrica, vol. 58, no. 6, pp. 1255-1277, Nov. 1990.

[8] G. Tan and J. Guttag, "The 802.11 MAC leads to Inefficient Equilibria," Proc. of IEEE INFOCOM 2005, pp. 1-11, March 2005.

[9] J. G. Proakis, Digital Communications, McGraw Hill, New York, 2000. 